\title{
Giant congenital melanocytic nevus coexisting with an asymmetric posture
}

\author{
Funda Tamer', Mehmet Eren Yuksel ${ }^{2}$
}

${ }^{1}$ Department of Dermatology, Ufuk University School of Medicine, Ankara, Turkey, ${ }^{2}$ Department of General Surgery, Aksaray University School of Medicine, Aksaray, Turkey

Corresponding author: Dr. Funda Tamer, E-mail: fundatmr@yahoo.com

\begin{abstract}
Congenital nevus is a melanocytic proliferation that is present at birth. When the diameter of a congenital melanocytic nevus is $20 \mathrm{~cm}$ or greater, it is called giant congenital melanocytic nevus. A giant congenital melanocytic nevus harbors the risk of malignant transformation. Moreover, the risk of malignant transformation increases with increasing lesion size. Furthermore, various abnormalities like scoliosis, spina bifida, atrophy and asymmetry of extremities can coexist with congenital nevi. Hereby, we present a 4-year-old Caucasian female child with a giant congenital melanocytic nevus on her trunk associated with postural asymmetry. The lesion depicted here had a characteristic clinical appearance, however the surface of the nevus had verrucous and hairy areas despite the patient's young age. The patient was evaluated for clinical signs of possible malignant changes, and regular dermatoscopic follow-up was recommended.
\end{abstract}

Key words: Congenital; Melanocytic nevus; Postural asymmetry

\section{INTRODUCTION}

A giant congenital nevus is a rare melanocytic lesion with an incidence of 1:20.000. It tends to be more common in women than men. It occurs during embryogenesis as a result of neuroectodermal abnormalities. Giant congenital melanocytic nevus clinically presents as an asymptomatic, brown-black colored, well defined lesion with hypertrichosis. Predilection sites are trunk, head and extremities. However, it can affect any part of the body surface [1].

Congenital nevi are classified according to their size. A congenital melanocytic nevus less than $1.5 \mathrm{~cm}$ in diameter is called small congenital melanocytic nevus. A congenital nevus with a size of between 1.5 $\mathrm{cm}-19.9 \mathrm{~cm}$ in diameter is called medium congenital melanocytic nevus. A giant congenital melanocytic nevus is greater than $20 \mathrm{~cm}$ in size. The patients with congenital melanocytic nevus should undergo regular clinical evaluation and dermatoscopic examination because of the risk of malignant transformation [2].

\section{CASE REPORT}

A 4-year-old Caucasian female child was admitted for further clinical evaluation of the hyperpigmented plaque on her trunk. The patient had this hyperpigmented lesion since birth and the lesion increased in size proportionally to the growth of her body. The lesion first appeared as a large brown patch with a smooth surface, however it became hairy and raised in time. Furthermore, an elevated lesion occured on the nevus within the last 6 months. The patient was otherwise healthy. She did not have any neurologic signs or symptoms. The family history was unremarkable. The dermatologic examination of the patient revealed a brown-black colored, hairy, round plaque with sharp borders which covered the neck, shoulders, right arm, chest, abdominal area and the upper back. The right lateral aspect of the lesion was ulcerated and elevated. In addition to the congenital nevus, multiple, small, hyperpigmented, round macules measuring $1 \mathrm{~mm}$ to $10 \mathrm{~mm}$ were observed on the trunk. Asymmetrical appearance of the neck, shoulders, and the trunk was 
noted (Figs. 1 and 2). Dermatoscopic examination revealed homogenous pigmentation, milia-like cysts, hair follicles and perifollicular hypopigmentation. Thus, the diagnosis of congenital melanocytic nevus was made based on the clinical and dermatoscopic features. A skin biopsy and histopathological examination of the ulcerated lesion were recommended to exclude a malignant transformation. Therefore, the patient was referred to the plastic and reconstructive surgery department.

\section{DISCUSSION}

Congenital nevus is a benign melanocytic lesion which presents at birth. However, $78 \%$ of the patients with giant congenital nevus also have small cutaneous hyperpigmented lesions which are called satellite lesions. Moreover, diffuse lipomatosis, cranial bone hypertrophy, limb atrophy, skeletal asymmetry, hyperplasia and hypoplasia of soft tissue, scoliosis, urinary tract anomaly, capillary malformation, café au lait spot, Mongolian spot and fibroepithelial polyp have been reported in patients with giant congenital melanocytic nevus previously [1]. In addition, Gorai et al. presented a giant congenital melanocytic nevus with occipital encephalocele in a 5-month-old male child in India [3]. Recently, Goyal et al. reported a 20-year-old female with a giant congenital nevus presenting as a large vulvar mass [4].

The risk of development of malignant melanoma in a giant congenital nevus varies from $1.8 \%$ to $45 \%$ [5]. Patients with the age of 3 to 5 years, patients with three or more lesions and lesions greater than $20 \mathrm{~cm}$ have higher risk of malignant transformation. A giant congenital nevus may become darker and thicker, moreover it can develop a papular, verrucous surface with time. However, it should be kept in mind that focal growth, ulceration, dark pigmentation, pruritus, pain and bleeding can be the warning signs of malignant transformation [5]. Furthermore, benign proliferative nodules which occur in congenital melanocytic nevi should be considered in differential diagnosis of malignant melanoma both clinically and histopathologically [6]. Su Han et al. reported a newborn with a giant congenital nevus with benign proliferative nodules on the genitalia which resembled a congenital malignant melanoma [7].

A giant congenital nevus may be associated with proliferation of melanocytes in the central nervous

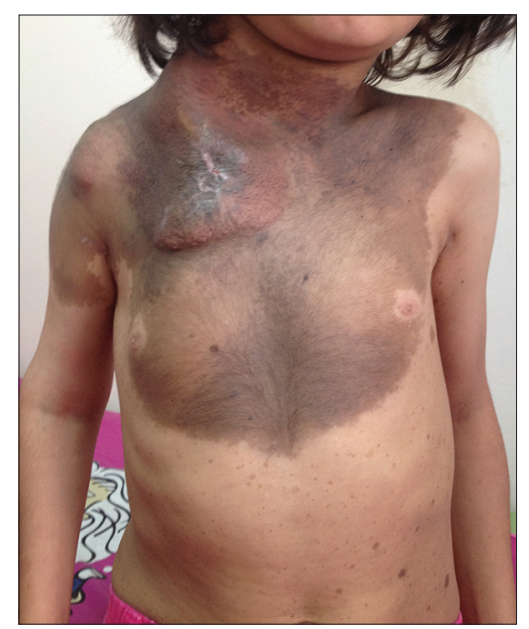

Figure 1: Well demarcated, oval, hairy, hyperpigmented plaque on the neck, chest, shoulders and right arm. Asymmetrical appearance of the trunk and atrophy of the upper extremities. Multiple hyperpigmented macules on the abdomen.

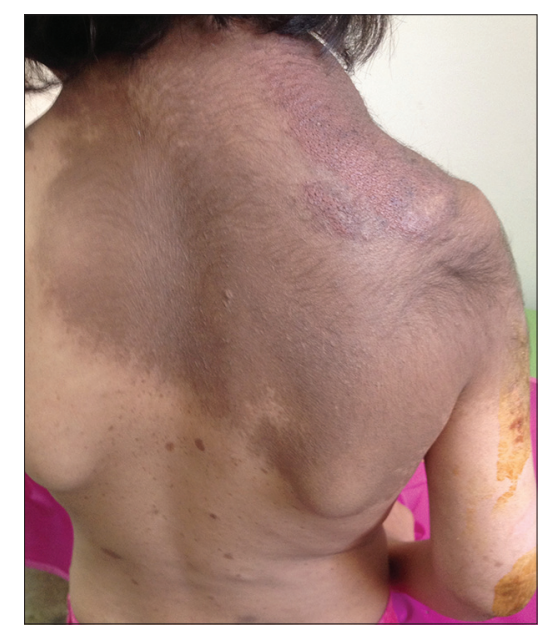

Figure 2: Well demarcated, oval, hyperpigmented plaque with brownorange colored plaques and hypertrichosis on the upper back. Multiple hyperpigmented macules on the lower back.

system which is called neurocutaneous melanosis. The risk of neurocutaneous melanosis in patients with giant congenital nevus is 3\%-12\%. Neurocutaneous melanosis may clinically present with increased intracranial pressure, seizures, motor deficits, myelopathy and radiculopathy [8].

Giant congenital nevi on the extremities can reduce the limb size as a result of infiltration of nevus cells in subcutaneous tissue [1]. Therefore, our patient had reduced diameter of the right upper extremity that caused an asymmetrical posture. However, our main concern was the possible risk of malignant transformation arising from the giant congenital nevus of the patient. 


\section{CONCLUSION}

In conclusion, a giant congenital melanocytic nevus harbors the risk of malignant transformation. Giant congenital nevi can lead to body shape disturbance even in childhood. Because of the risk of malignant transformation, giant congenital nevi should be carefully evaluated.

\section{REFERENCES}

1. Viana AC, Gontijo B, Bittencourt FV. Giant congenital melanocytic nevus. An Bras Dermatol. 2013;88:863-78.

2. Tannous ZS, Mihm MC Jr, Sober AJ, Duncan LM. Congenital melanocytic nevi: Clinical and histopathologic features, risk of melanoma, and clinical management. J Am Acad Dermatol. 2005;52:197-203.

3. Gorai S, Saha M, Seth J. Giant congenital melanocytic nevus with occipital encephalocele: A very rare association. Indian J Dermatol. 2016;61:224-6.
4. Goyal M, Kriplani A, Mahey R, Kachhawa G, Yadav R, Singh G. Giant congenital melanocytic nevus presenting as a huge vulvar mass: A rare case. Taiwan J Obstet Gynecol. 2017;56:109-11.

5. Arneja JS, Gosain AK. Giant congenital melanocytic nevi. Plast Reconstr Surg. 2009;124:1e-13e.

6. Vergier B, Laharanne E, Prochazkova-Carlotti M, de la Fouchardière A, Merlio JP, Kadlub N, et al. Proliferative nodules vs melanoma arising in giant congenital melanocytic nevi during childhood. JAMA Dermatol. 2016;152:1147-51.

7. Han JS, Won CH, Chang SE, Lee MW, Choi JH, Moon KC. Giant congenital melanocytic nevus with proliferative nodules mimicking a congenital malignant melanoma. Ann Dermatol. 2014;26:554-6.

8. Sawicka E, Szczygielski O, Żak K, Pęczkowski P, Michalak E, Bekiesińska-Figatowska M. Giant congenital melanocytic nevi: Selected aspects of diagnostics and treatment. Med Sci Monit. 2015;21:123-32.

Copyright by Funda Tamer, et al. This is an open-access article distributed under the terms of the Creative Commons Attribution License, which permits unrestricted use, distribution, and reproduction in any medium, provided the original author and source are credited.

Source of Support: Nil, Conflict of Interest: None declared. 